Session 2559

\title{
Killing Two Birds with One Data Acquisition System
}

\author{
James E. Mayhew, Richard A. Layton \\ Rose-Hulman Institute of Technology
}

\begin{abstract}
An open-circuit wind tunnel is upgraded by adding a commercially-available data acquisition system used to teach students some basic concepts of data acquisition, instrumentation, calibration, and assessment of results. Student teams were given 30-60 minutes of hands-on instruction on how to acquire data using the system. Eight student teams participated over two quarters, performing calibrations of the load cells and angle-of-attack indicator, using the results of those calibrations to find the lift and drag of a model wing, and assessing whether the calibrations and confidence intervals found by the earlier teams were reliable. All teams served as "contractors" for us, helping us improve the quality of our wind tunnel while they learned. Key results for our students: learning how to set up and use a simple data acquisition system; making us aware of sources of uncertainty in the lift and drag measurements of our wind tunnel; learning when collecting more data helps decrease uncertainty and when it does not; and gaining experience in meeting our needs as customers. In our opinion, the project is readily implemented by an individual instructor or two and should be considered intermediate-level instruction in instrumentation and data acquisition, appropriate for implementation at the junior or senior level.
\end{abstract}

\section{Introduction}

Upgrading undergraduate labs to incorporate modern, computer-based instrumentation and data acquisition is a common area of continuous improvement in engineering programs as faculty work to provide students with instruction in this technology. Upgrades can include the improvement of a single apparatus such as the heat exchanger upgrade described by Hinton et al. ${ }^{1}$ or the wind tunnel upgrade described in this paper, or the upgrade of several experiments within a lab such as that described by Craig and McConnell ${ }^{2}$, or the development of a new laboratory as described by Knight and McDonald ${ }^{3}$, to the integration of instrumentation skills across several courses and disciplines such as that described by Mahajan and others. ${ }^{4,5}$ While the work described in this paper is modest compared to program-wide innovative developments described by some of these authors, the data-acquisition application we present is a low-cost approach to giving students a valuable learning experience with an open-ended problem while enhancing our knowledge of wind tunnel experimental results with a not-too-steep learning curve for the students, the faculty, and our lab technicians.

At Rose-Hulman Institute of Technology, in years past, mechanical engineering students performed a conventional experiment using our wind tunnel as part of a senior lab course. The objective was to find lift and drag polars for a scale-model wing. Students had no control over 
the data acquisition setup. They gained experience in operating the wind tunnel, but they did not develop a sense of whether the data were reliable. This bred an "it came from a computer so it must be right" attitude. While the overall quality of the wind tunnel itself was excellent, the random variations in lift and drag readings seemed unusually large. Nevertheless, most students were motivated about doing the experiment even while they complained in their course evaluations about using "antiquated" equipment.

The old data acquisition software, developed in-house, was running on an IBM-compatible 386 computer and was incompatible with the newer Windows computer we had purchased as part of general lab upgrade. Moreover, our department had recently purchased a new Agilent data logger for general lab use, and our electronics technician was eager to have someone try it out. We decided if we could get the system acquiring data-any data-within 60 minutes of opening the box in which it was packaged, then we could expect the students to learn it quickly enough to setup and use in a lab experiment. (We did and they could.) To take advantage of some of the features of the new data acquisition system, we developed some new lab experiments that were more open-ended than the conventional wind-tunnel experiments we had done in years past. One added benefit to our students of these experiments is that the experiments require the student to exercise the higher-level thinking skills of synthesis (design, construct, and/or improve an existing apparatus) and evaluation (critique, justify, and assess experimental results). ${ }^{6}$

\section{Objectives and Course Description}

The main learning objectives ("bird" \#1) for the students of these mini-projects are:

1. To help students learn how to use a data acquisition system.

2. To help students learn to assess the reasonableness of wind tunnel experimental results that they developed themselves as well as results produced by others.

The main objectives ("bird" \#2) for the faculty as customers of these mini-projects are:

1. Enhance the wind tunnel lab experience for our students.

2. Improve our knowledge of wind tunnel performance.

Students performing the experiments (or mini-projects) described in this paper are enrolled in a required senior laboratory course in mechanical engineering. The course has one hour of lecture and three hours of lab per week for ten weeks for 2 credit-hours. In this course, ME421, students perform two lab experiments over six weeks plus one 10-week project involving some aspect of experimental design. The mini-projects described in this paper were assigned as one of the two experiments, not the project.

We think the way we supervised these projects is new and interesting to others because it forced the students to simulate an engineer's role in an industrial environment, where one doesn't know the results beforehand and must try to make sense out of the results one does get. In addition, subsequent teams had the enlightening and often frustrating experience of reading, interpreting, and deciding whether or not to trust the reports produced by prior teams. 


\section{System Overview}

The wind tunnel used in these experiments is an open-circuit, low-speed type with a nominal test section size of 12 x 17 inches, manufactured by Aerolab. ${ }^{7}$ In a conventional experiment, a model airfoil is mounted inside the test section to a pyramidal balance which transmits lift and drag forces to load cells. The load cell output signals are conditioned by a factory-provided signal conditioner producing voltage signals (in the millivolt range) proportional to lift and drag forces. Pitch moment is also measured, but is not used in this experiment. Angle of attack is varied manually using a worm gear linkage provided with the pyramidal balance.

By measuring air pressure and temperature, airfoil planform area, air velocity, and the lift and drag forces, lift and drag coefficients can be determined as a function of angle of attack. The resulting lift and drag curves are familiar to students in aerodynamics courses, and if accurate can be used in aircraft design. One of the goals of our lab upgrade is to eventually enable students to obtain experimental results comparable to published NACA data, such as that available for the Clark Y airfoil. ${ }^{8}$

\section{Experimental Setup}

Data Acquisition System. The new data logger is an Agilent 34970A Data Acquisition/Switch Unit (DAS). New small-gage conductors from the existing wind tunnel signal conditioning unit are manually attached to the screw terminals of a multiplexer (MUX) module, ordered with the DAS, which plugs directly into the back of the DAS. An RS232 cable connects the DAS to a 529 $\mathrm{MHz}$, Intel X86 running Windows NT2000. The software ordered with the DAS and installed on the PC is Agilent Benchlink Data Logger.

Wind Tunnel Setup. The experimental setup varied depending on the task the student team was assigned. The team selected on experiment from gave a menu of experiment descriptions we provided. Experiments were intended to span about ten hours of the team's time, for teams of four students. Possible tasks included:

- fabricate a Pitot tube to correlate true airspeed to corrected airspeed.

- definitively calibrate the lift load cell.

- definitively calibrate the drag load cell.

- definitively calibrate the angle of attack indicator.

- obtain a lift or drag curve for a given airfoil, checking that the results are consistent with reported confidence intervals.

In the case of the calibration experiments, students did not have to operate the wind tunnel, but did have to design and fabricate an apparatus for performing their calibration.

A thermocouple was inserted in the test section for temperature measurements used to correct for non-standard-temperature-density test conditions.

Data Acquisition System Setup. Four data channels were connected from the wind tunnel instrumentation to the data acquisition system; lift force, drag force, pitch moment, and air 
temperature. Students used the data acquisition ssoftware to control which channels were sampled, to set sample rates, and to peform data analysis. Some of the features of the Agilent Data Logger that helped make the experiment successful are briefly discussed below.

A 16-Channel Multiplexer. Most of our students had heard of a multiplexer before, but none had ever held one in their hands. Showing them the simple plug-in module took a lot of the mystery out of this device.

Trace of Data like an Oscilloscope. The software that comes with the Data Logger shows a trace on the computer screen for each channel acquiring data. Further, the data acquisition box had a real-time digital readout, very much like a multi-channel multimeter. An example screen-shot is in Figure 1. In this case, both drag and temperature measurements are shown on the same oscilloscope-like trace. Channels can be added or de-selected, and scales can be auto-set so that measurements with different amplitude scales can be shown on the same plot.

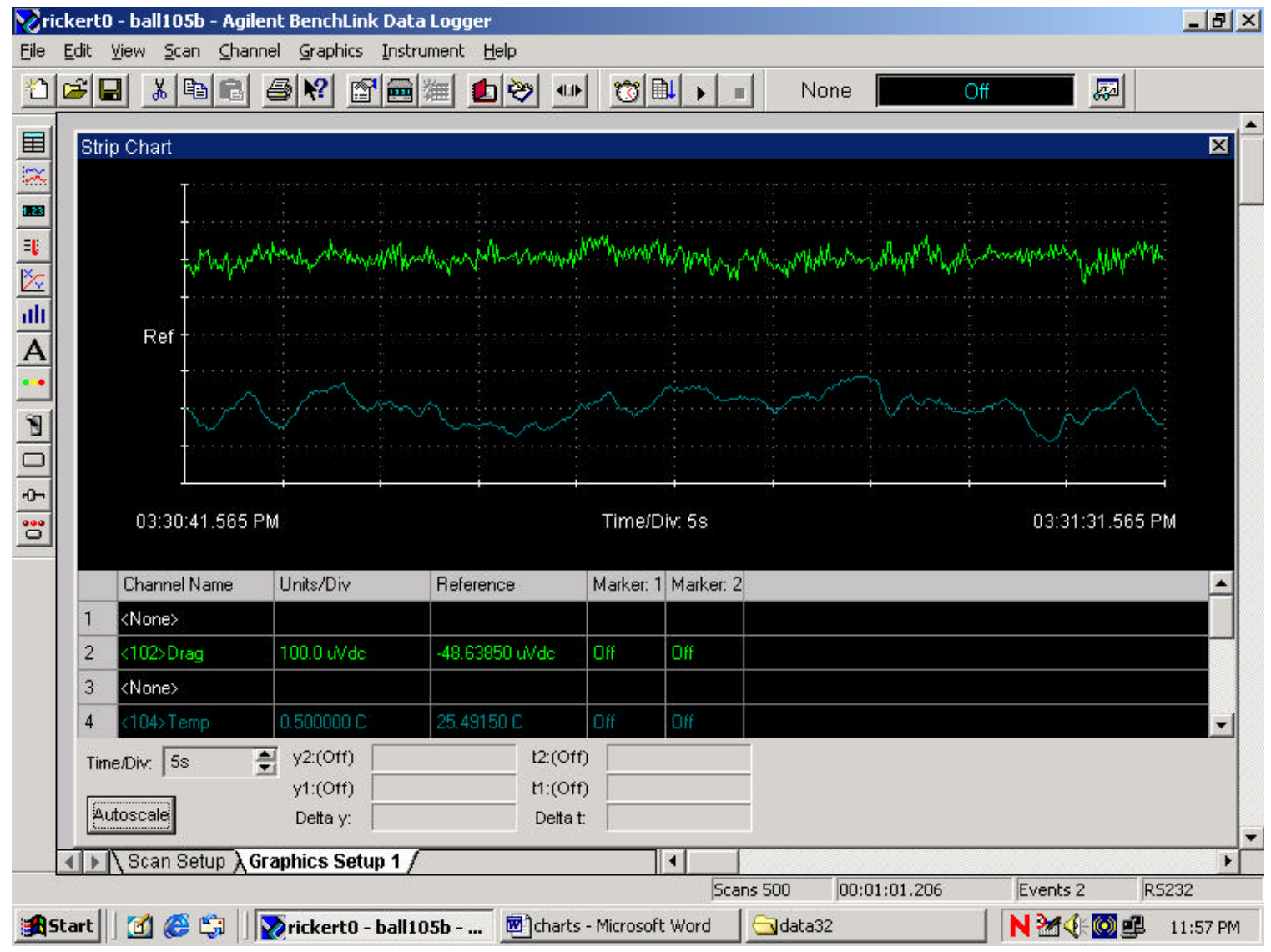

Figure 1: Sample drag and temperature measurements on the computer screen. 


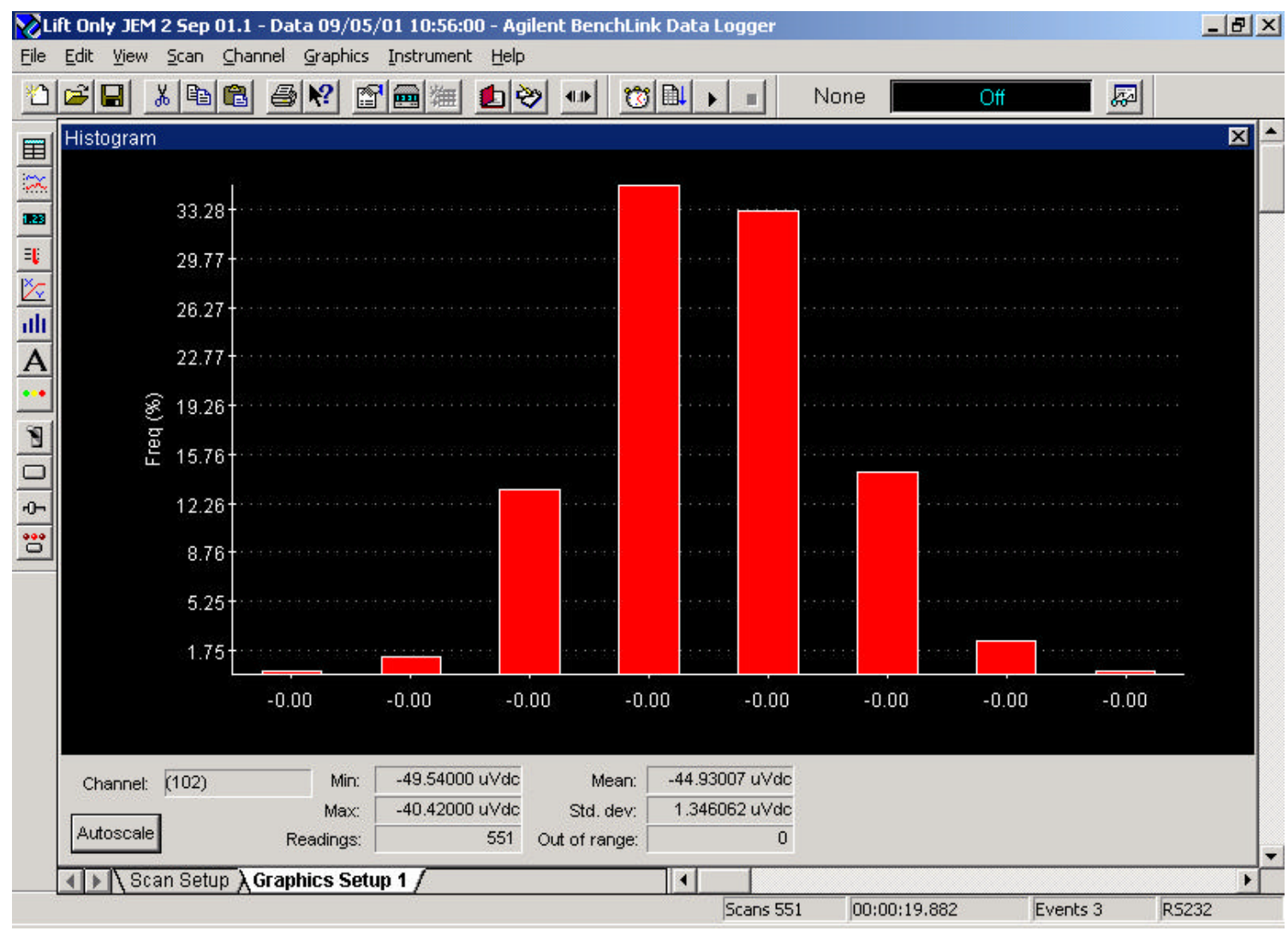

Figure 2: Sample histogram of lift measurements.

Histogram. A histogram of the data being acquired can be displayed at near real time, and it shows the number of samples taken and the mean and standard deviation being updated with each new sample. An example of the historam feature is shown in Figure 2. 


\begin{tabular}{|c|c|c|c|c|c|c|c|c|c|c|c|c|c|c|}
\hline \multicolumn{13}{|c|}{ Rlayton trial - Data 03/14/02 23:43:04 - Agilent BenchLink Data Logger } & \multicolumn{2}{|c|}{- 可x } \\
\hline \multicolumn{15}{|c|}{ File Edit Yiew Scan Ghannel Graphics Instrument Help } \\
\hline 3 & {$[\tilde{E} \mid \mathbb{P}$} & 1) & |跑|술 & 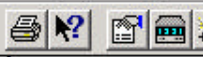 & 且安 & 415) & 3)趾 & $\square$ & None & & Off & 圈 & & \\
\hline & \multicolumn{3}{|c|}{ Channel } & \multicolumn{4}{|c|}{ Measurement } & \multicolumn{4}{|c|}{ Scaling $(M x+B)$} & \multicolumn{3}{|r|}{$\triangle$} \\
\hline 贯 & ID & Sca & Name & Function & Range & Resolutio & Advance & Scale & Gain(M) & Offsetl & Label & Test & High & \\
\hline 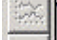 & 101 & $\sqrt{\square}$ & Lift & DC volts & Auto & 5.5 digits & Default & $\Gamma$ & 1.0 & 0.0 & VDC & $\overline{O f f}$ & 0.0 & \\
\hline 표 & 102 & $\sqrt{\nabla}$ & Drag & DC volts & Auto & 5.5 digits & Default & $\Gamma$ & 1.0 & 0.0 & VDC & Off & 0.0 & \\
\hline El & 103 & $\nabla$ & Pitch & DC volts & Auto & 5.5 digits & Default & $\Gamma$ & 1.0 & 0.0 & VDC & Off & 0.0 & \\
\hline $\bar{x}$ & 104 & $\bar{V}$ & Temp & Temp (type K) & & & Default & $\Gamma$ & 1.0 & 0.0 & C & Off & 0.0 & \\
\hline ili & 105 & $\Gamma$ & & DC volts & Auto & 5.5 digits & Default & $\Gamma$ & 1.0 & 0.0 & VDC & Off & 0.0 & \\
\hline $\mathrm{A}$ & 106 & $\Gamma$ & & DC volts & Auto & 5.5 digits & Default & $\Gamma$ & 1.0 & 0.0 & VDC & Off & 0.0 & \\
\hline$\ldots$ & 107 & $\Gamma$ & & DC volts & Auto & 5.5 digits & Default & $\Gamma$ & 1.0 & 0.0 & VDC & Off & 0.0 & \\
\hline- & 108 & $\Gamma$ & & DC volts & Auto & 5.5 digits & Default & $\Gamma$ & 1.0 & 0.0 & VDC & Off & 0.0 & \\
\hline$\Phi$ & 109 & $\Gamma$ & & DC volts & Auto & 5.5 digits & Default & $\Gamma$ & 1.0 & 0.0 & VDC & Off & 0.0 & \\
\hline$\square$ & 110 & $\Gamma$ & & DC volts & Auto & 5.5 digits & Default & $\Gamma$ & 1.0 & 0.0 & VDC & Off & 0.0 & \\
\hline (2)- & 111 & $\Gamma$ & & DC volts & Auto & 5.5 digits & Default & $\Gamma$ & 1.0 & 0.0 & VDC & Off & 0.0 & \\
\hline \multirow[t]{6}{*}{ 骂 } & 112 & $\Gamma$ & & DC volts & Auto & 5.5 digits & Default & $\Gamma$ & 1.0 & 0.0 & VDC & Off & 0.0 & \\
\hline & 113 & $\Gamma$ & & DC volts & Auto & 5.5 digits & Default & $\Gamma$ & 1.0 & 0.0 & VDC & Off & 0.0 & \\
\hline & 114 & $\Gamma$ & & DC volts & Auto & 5.5 digits & Default & $\Gamma$ & 1.0 & 0.0 & VDC & Off & 0.0 & \\
\hline & 115 & $\Gamma$ & & DC volts & Auto & 5.5 digits & Default & $\Gamma$ & 1.0 & 0.0 & VDC & Off & 0.0 & \\
\hline & 116 & $\Gamma$ & & DC volts & Auto & 5.5 digits & Default & $\Gamma$ & 1.0 & 0.0 & VDC & Off & 0.0 & \\
\hline & \multicolumn{14}{|c|}{$\Delta D$ Scan Setup A Graphics Setup $1 /$} \\
\hline Activa & atejDeac & tivate c & hannel & & & & & HP34902 & & one) & & & $\mathrm{RS} 232$ & \\
\hline
\end{tabular}

Figure 3: Data logger channel scan setup screen.

Simple Scan Setup. The setup was all Windows-driven and was intuitive. The sampling rate and number of samples could easily be changed, as could the channels being sampled. The setup of each channel is handled via the software as shown in Figure 3.

\section{Procedure}

The first two student teams began in the Fall 2001-2002 quarter. We told them they were like contractors that we hired to get the wind tunnel into good enough operating condition so that we could offer it to off-campus customers for research—to sell wind tunnel time.

The problem statement for the lab exercises for these first two teams was as follows:

- Calibrate the load cells to relate voltage output to lift and drag force

- Fabricate necessary hardware to perform the calibration procedure and data collection

- Use the data acquisition system to reduce random uncertainty in measurements

- Measure the lift and drag of a wing model in the wind tunnel

The first group had to calibrate the lift load cell, and the second group had to calibrate the drag load cell. For the second group this included designing and building an apparatus to perform the calibration (we already had an apparatus for calibrating the lift load cell). Since we had the new 
data acquisition system, we expected this calibration to include confidence intervals with a good documentation of the statistics work done. In addition, they were to check their work by testing a wing model in the tunnel and measuring lift (Team 1) and drag (Team 2). They had three weeks to complete this task.

On their first day in the lab, we gave them 30 to 60 minutes of instruction on how to use the wind tunnel and the data logger. After that, we helped them only when they asked. This ranged from giving advice about how to build the drag calibration device to choosing an appropriate sample size for the data collection.

After the first two teams were done, we "hired" another two student teams to serve as our "contractors," but this time they were to play the role of external customers who were paying to use our wind tunnel. Their charge was to find the lift and drag coefficients, respectively, on a wing model using the calibration curves the first two groups had produced, and assess whether the results were reliable. In particular, did the confidence intervals for the calibration curves seem realistic?

The role of the instructors in this laboratory course was not limited to giving advice. Perhaps our most important function was monitoring the teams' progress and making sure they kept up the pace to finish in the required three weeks. Further, we were always thinking at least one project ahead; that is, as we saw the students getting results we knew we wouldn't be satisfied with in the long term, we devised new projects for the next group of students focusing on those trouble areas. For example, the following quarter (Winter 2001-2002) we offered the course again, with the following problem statement:

- Perform a definitive calibration of the lift load cell.

- Include a report on the effects of hysteresis, dead zone, and repeatability.

- Fabricate new hardware to apply upward and downward lift loads.

- $\quad$ Produce a calibration curve with a prediction interval.

For the later teams doing calibrations, both a calibration curve and a calibration report modeled on Instrument Society of America standards were required, producing percentage estimates of accuracy, repeatability, and hysteresis plus deadband. ${ }^{9}$ The standard to which we held the later groups was therefore higher, but the scope of the project narrower. This is consistent with our long-range goal of improving the quality of the wind tunnel operating characteristics.

\section{Results}

Student Expermental Results. The first two teams presented us with calibration curves as required, but with weak uncertainty analyses. Their confidence intervals were not well justified. They had spent most of their time thinking about the calibration procedure itself, and in the case of team 2, designing and building the calibration apparatus. They also recommended several areas for future work, some of which we had not considered before.

Although we did not get definitive calibration curves like we wanted, we were pleased that the students were forced to think through the calibration process, and were able to acquire 
calibration data and present us curves, even if the uncertainty analyses were incomplete. It was clear that we had given them the tools to solve similar problems in industry, and that they would soon see firsthand that their uncertainty analysis was lacking. In hindsight, we realized that the first two experiments assigned were too long for the allotted time. Building an apparatus, conducting a calibration, and running an experiment all for the first time could not be done well in 3 weeks.

The second two teams found that the first two teams did not give them reliable calibration results. Although they successfully produced plots of lift and drag coefficient, they did not compare well with published data, and their measurements were outside of the confidence intervals quoted by the first two groups. In short, they would have been extremely dissatisfied customers if they had been paying for the tunnel time and expecting to have reliable calibration curves.

Teams 3 and 4 used the reports from teams 1 and 2. They were able to see how the discussions were vague and found errors. More areas for further work became apparent to us all, including pointing out that the lift calibration had been done by applying a force in a downward direction, but during tunnel operation the lift force is usually in an upward direction. Also, the mechanism for setting and measuring angle of attack for the wing had a substantial amount of hysteresis and deadband, resulting in poor repeatability.

Summary: we still don't have a definitive calibration of the lift and drag measurements in our wind tunnel, but the students have identified the areas that need attention in order to get it.

The Educational Results. Students learned to use the data acquisition system quickly, allowing them to focus on making low uncertainty measurements. They spent their time on fundamental concepts (atmospheric vs. stagnation pressure, computing lift and drag coefficients, etc.), not regarding the data acquisition system. In particular, the statistical features of the software were helpful and easy to use.

Students were able to apply their statistics skills and see how larger sample sizes reduce the random uncertainty. With one group we spent 3 to 5 hours over several meetings discussing what sample size would be large enough to have a negligible random uncertainty, but as small as possible in order to save time in data collection. This was a level of subtlety we had never reached with students in this course in the past.

Students realized the importance of investing the effort to understanding prior work on a similar project. Unfortunately, they typically realized this too late in the project timeline. Although teams that followed up to verify prior work did not generally do a good job of understanding the prior work in depth sufficient for their needs, they did eventually realize the importance of deep understanding of prior work.

Focusing each lab on a specific, definitive report with the goal of placing the student report into the permanent record leading to the "certification of the wind tunnel for commercial use" provided good student motivation. 
Summary: The use of a user-friendly data acquisition system allowed our students to spend their time on issues related to reducing uncertainty in measurements. It also allowed them to learn about instrumentation systems generally.

\section{Conclusions}

The Agilent Data Logger data acquisition system allowed students unfamiliar with data acquisition to confidently acquire lift and drag data in a wind tunnel and analyze its quality.

Students will not produce "definitive" calibrations the first or second time through a calibration procedure. However, they will probably identify problems with the procedure that must be corrected before getting the definitive result.

Students can do a problem similar to what other students have already done, and look at their report, but still learn from the experience. This is a realistic situation in engineering practice, where others' results may be available and should be consulted, but should not necessarily be trusted. Students will likely resist giving a lot of attention to the previous work, thinking it a waste of time.

We have begun a permanent record of the operating characteristics for our tunnel. To date this record includes six student reports completed over a span of three months.

Undergraduate students can be used to document and improve the operating characteristics of laboratory equipment. We have another wind tunnel needing the same types of calibrations, and plan to use a similar approach with it as well as other laboratory equipment with unknown operating characteristics.

Once basic calibrations are complete, we can move on to definitive use of the wind tunnels to obtain lift and drag polars, and compare corrected results to published data for the same airfoils at the same operating conditions. We can then periodically return to the experiments described above to verify the performance of the lift and drag measurement system.

Bibliography

1. Hinton, M.A., Marloth, R., and Noorani, R. (2000). Data acquisition system for an undergraduate fin heat exchanger experiment. In proc. ASEE Annual Conference. ASEE, St. Louis, June 2000.

2. Craig, R.R., Jr. and McConnell, E.L. (1999). Virtual instruments revitalize an undergraduate measurements and instrumentation course. In proc. ASEE Annual Conference. ASEE, Charlotte, June 1999.

3. Knight, C.V. and McDonald, G.H. (1998). Modernization of a mechanical engineering laboratory using data acquisition with LabVIEW. In proc. ASEE Annual Conference. ASEE, Seattle, June 1998.

4. Mahajan, A., Walworth, M., McDonald, D., and Schmaltz, K. (1999). The integrated systems engineering laboratory - an innovative approach to vertical integration using modern instrumentation. In proc. ASEE Annual Conference. ASEE, Charlotte, June 1999.

5. McDonald, D., Adams, R., Duesing, P., and Mahajan, A. (1997). Improving the laboratory experience with modern computer-based instrumentation. In proc. ASEE Annual Conference. ASEE, Milwaukee, June 1997. 
6. Felder, R.M. and Brent, R. (1999). How can I teach students to be effective problem-solvers and to exercise higher-level thinking skills? In handbook, National Effective Teaching Institute. ASEE, Charlotte, June 1999.

7. URL: http://www.aerolab.com/. Aerolab, Laurel, Md, 1997.

8. Higgins, G.J. (1927). The characteristics of the NACA 97, Clark Y, and NACA-M6 airfoils with particular reference to the angle of attack. NACA TN 270. Available at URL: http://naca.larc.nasa.gov/

9. Process Instrumentation Terminology Standard (1979). ANSI/ISA-S51.1-1979. Instrument Society of America, Research Triangle Park, NC.

\section{JAMES E. MAYHEW}

James E. Mayhew received his Ph.D. from the University of California, Davis in 1999. He is currently an Assistant Professor of Mechanical Engineering at Rose-Hulman Institute of Technology. Prior to this, Dr. Mayhew was a US Air Force Officer, working in research, development, and logistics engineering for 6 years and teaching aeronautics at the US Air Force Academy for 5 years.

\section{RICHARD A. LAYTON}

Richard A. Layton received his Ph.D. from the University of Washington in 1995 and is currently an Assistant Professor of Mechanical Engineering at Rose-Hulman Institute of Technology. Prior to his academic career, Dr. Layton worked in industry for twelve years, culminating as a technical group head and a project manager. He is a registered Professional Engineer, chair of the Modeling and Identification Technical Panel of the Dynamic Systems and Control Division of ASME, and an active member of the Educational Research and Methods Division of ASEE. 